\title{
Carcinoid tumour of the thymus with systemic manifestations: a radiological and pathological study
}

\author{
R. M. L O W ENTHA L ${ }^{1}, J$. M . GUMPEL, L. KREEL, \\ J . E. M c LAUGHLIN ${ }^{2}$, a nd D. B. L. SKEGGS \\ Departments of Medicine and Radiology, Northwick Park Hospital, Watford Road, \\ Harrow, Middlesex; and Departments of Pathology and Radiotherapy, Royal Free Hospital, \\ North-Western Branch, Lawn Road, London NW3
}

Lowenthal, R. M., Gumpel, J. M., Kreel, L., McLaughlin, J. E., and Skeggs. D. B. L. (1974). Thorax, 29, 553-558. Carcinoid tumour of the thymus with systemic manifestations: a radiological and pathological study. Following recent reports of an unusual mediastinal tumour described as 'mediastinal endocrine neoplasm of probable thymic origin, related to carcinoid tumour' (Rosai and Higa, 1972), a further case, in a 72-yearold man, has been studied.

Polyarthropathy was the presenting feature, and the patient also had clubbing of the fingers and clinical evidence of a probable proximal myopathy and a peripheral neuropathy. These non-metastatic systemic manifestations have not previously been described with this type of tumour.

By retrograde thymic venography the tumour was conclusively shown to be arising in the thymus, as had been suspected but not proven in previously described cases. Histologically the typical picture, including areas of cells with true rosette formation and cells with argyrophil granules, was seen.

In contrast to the more usual thymic tumours, this type of neoplasm responds poorly to radiotherapy, but surgical treatment may give good results. Therefore, in a situation where the possibility of carcinoid tumour of the thymus exists, it is imperative that tissue be obtained for histological diagnosis before any therapeutic decision is made.

Recent reports have drawn attention to an unusual type of primary mediastinal tumour, histologically resembling carcinoid tumours in other sites (Rosai and Higa, 1970; Rosai and Higa, 1972). We report here the clinical and pathological details of a case in which a number of previously unreported non-metastatic systemic manifestations were present, and in which the location of the tumour in the thymus was confirmed by radiological techniques.

\section{CASE REPORTS}

A man aged 72 presented with a nine-month history of gradually worsening polyarthritis with pain and swelling affecting particularly the wrists, hands, hips,

${ }^{1}$ Present address and address for correspondence: MRC Leukaemia Unit, Royal Postgraduate Medical School, Du Cane Road, London W12 OHS

'Present address: Institute of Pathology, The London Hospital, London E1 and knees, and with paraesthesiae in the fingers. For two months he had experienced difficulty in holding cups and in raising himself from the sitting position. For a few weeks he had had malaise, anorexia with slight weight loss, and dyspnoea on climbing stairs. He was a pipe smoker consuming two ounces of tobacco per week. Six months previously he had undergone a routine herniorrhaphy; at that time no systemic disease had been suspected.

He was an ill-looking man with prominent finger clubbing. Wrist extension was limited to $50^{\circ}$ bilaterally and the metatarsophalangeal joints were tender. There was clear evidence of free synovial fluid in both knees. The heart rate was $72 / \mathrm{min}$ with-from time to time-the characteristics of pulsus bigeminis. The blood pressure was $170 / 110 \mathrm{mmHg}$. There was a harsh systolic ejection-type murmur heard maximally in the second left intercostal space anteriorly. The liver was firm and enlarged, the edge being $5 \mathrm{~cm}$ below the right costal margin. Neurological examination suggested a mild proximal myopathy with weakness 
of the deltoids, left triceps, and trunk and thigh muscles. There was also slight blunting of light touch sensation on the fingers.

The haemoglobin was $13.4 \mathrm{~g} / 100 \mathrm{ml}$, the white blood count was normal, and the erythrocyte sedimentation rate $14 \mathrm{~mm}$ in one hour. Routine biochemical estimations, including blood urea and serum electrolytes, serum proteins, serum alkaline phosphatase, serum calcium and phosphate, and serum uric acid, were all normal. Cytological examination of the sputum on several occasions revealed no malignant cells. Antinuclear and thyroid microsomal antibodies were present in low titre in the serum $(1: 10)$; tests for other tissue antibodies were negative. The electrocardiogram (ECG) showed frequent atrial ectopic beats; this dysrhythmia was intermittent. A phonocardiogram showed a late systolic murmur spilling over the aortic second sound (Fig. 1), thus indicating that it was arising from the pulmonary region.

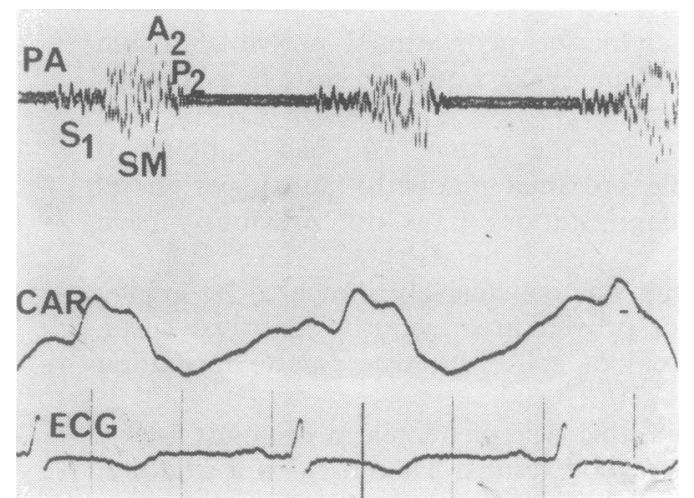

FIG. 1. Phonocardiogram from pulmonary area $(P A)$ showing an ejection-type late systolic murmur (SM) spilling over the aortic second sound. $S_{1}$, first heart sound; $A_{2}$, aortic, and $P_{2}$, pulmonary component of the second sound; CAR, carotid trace; ECG, electrocardiogram.

The chest radiograph (Fig. 2) revealed a left-sided anterior mediastinal mass, accentuated by the right anterior oblique position, its lower margin forming a slight angle with the left heart border. The mass was closely applied to the main pulmonary artery and, in appearance and its position, was highly suggestive of a left-sided thymoma. In view of the negative sputum cytology it was considered important to show whether the tumour was within the thymus or whether a normal thymus was being displaced by the mass. Retrograde thymic venography confirmed the presence of a thymic tumour with a very vascular proximal portion. An interesting feature was the outlining of an anterior aortic venous plexus from the injection of the thymic vein (Fig. 3).

After only a few days in hospital, comparison with an earlier radiograph showed an extremely rapid increase in the size of the tumour. An urgent transfer of the patient for radiotherapy was therefor arranged.

Cobalt teletherapy was given by a multiple fie technique. After 11 days, when the tumour haf received an effective dose of 1150 rads, the patient developed cardiac failure with a pericardial rub and a large left pleural effusion. He was given digitalis and diuretics, and radiotherapy was suspended. The effusion was tapped twice. No malignant cells wee identified in the straw-coloured fluid. After two weels his condition had improved sufficiently to allow radip therapy to be resumed, and a total modal dose $O P$ 4,000 rads was reached 53 days after the start of treatment. The tumour regressed only slight (original treatment field $15.5 \times 17 \mathrm{~cm}$; final fietr $15.5 \times 13.5 \mathrm{~cm})$. There was brief clinical improve ment, but eight weeks after the end of therapy he was readmitted to hospital almost moribund. Thf liver was massively enlarged and a metastatic ski nodule was identified over the left loin. He died the following day.

PATHOLOGICAL FINDINGS Necropsy revealed a tumour in the anterior mediastinum invading the parietal pericardium posteriorly but not involving viscera pericardium or myocardium, and the left luns laterally, which on section presented a homogeneous white cut surface interspersed with small areas of necrosis. A $5 \mathrm{~cm}$ diameter haemorrhagic nodule $w$ present on the posterolateral aspect of the left parieta pleura inferiorly. Other tumour deposits were foung in the right lobe of the liver (weight 3,000 g), the leff lobe of the thyroid, the left adrenal (weight $65 \mathrm{~g}$ the right adrenal (weight $10 \mathrm{~g}$ ), and in the subcutaneous tissue of the left loin. No abnormality was seen on dissection of the bronchial tree, and the pituitary, pancreas, gastrointestinal tract, and testes were all unremarkable. No parathyroids were ident's? fied. The heart showed myocardial hypertrophy, but no valve lesion was seen and the endocardial surfaces appeared normal.

The gross appearances suggested a diagnosis malignant thymoma with multiple metastases.

Microscopy revealed a tumour composed relatively uniform round or polygonal cells of medium size with central vesicular nuclei and eosing philic cytoplasm. Areas composed of tall cells showing rosette formations were prominent (Fig. 4). The luminal border of these cells appeared as a distind eosinophilic membrane and a little weakly PAS5 positive material was present in the lumen. N\& mucin production could be demonstrated. Much necrosis and moderate numbers of mitoses were seeno Staining for argentaffin granules by the diazo and Masson Fontana technique was negative but argyrophil granules could be demonstrated by the-Bodia method (Fig. 5). A Congo red stain for amyloi was negative. Electron microscopic examination o the formalin-fixed postmortem material after posto fixation in osmic acid and staining with urany 


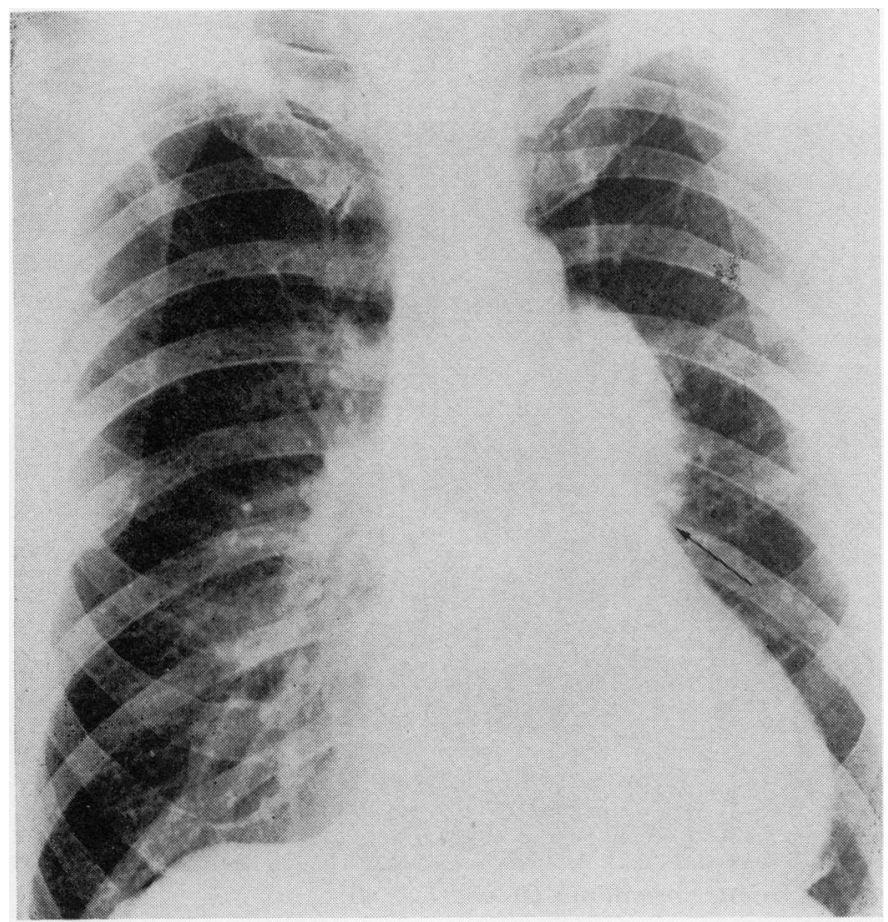

FIG. 2. Postero-anterior chest radiograph on admission demonstrating a left-sided anterior mediastinal tumour.

FIG. 3. Retrograde flow of contrasi medium from thymic plexus demonstrates a peri-aortic venous plexus.

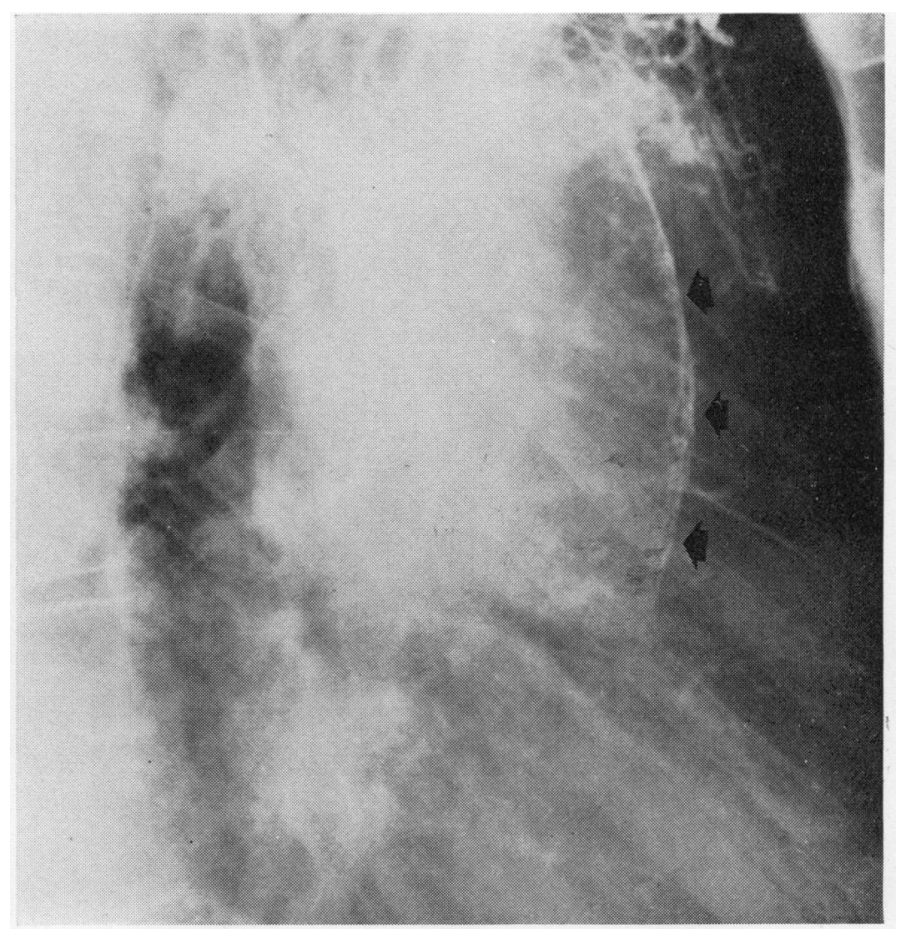




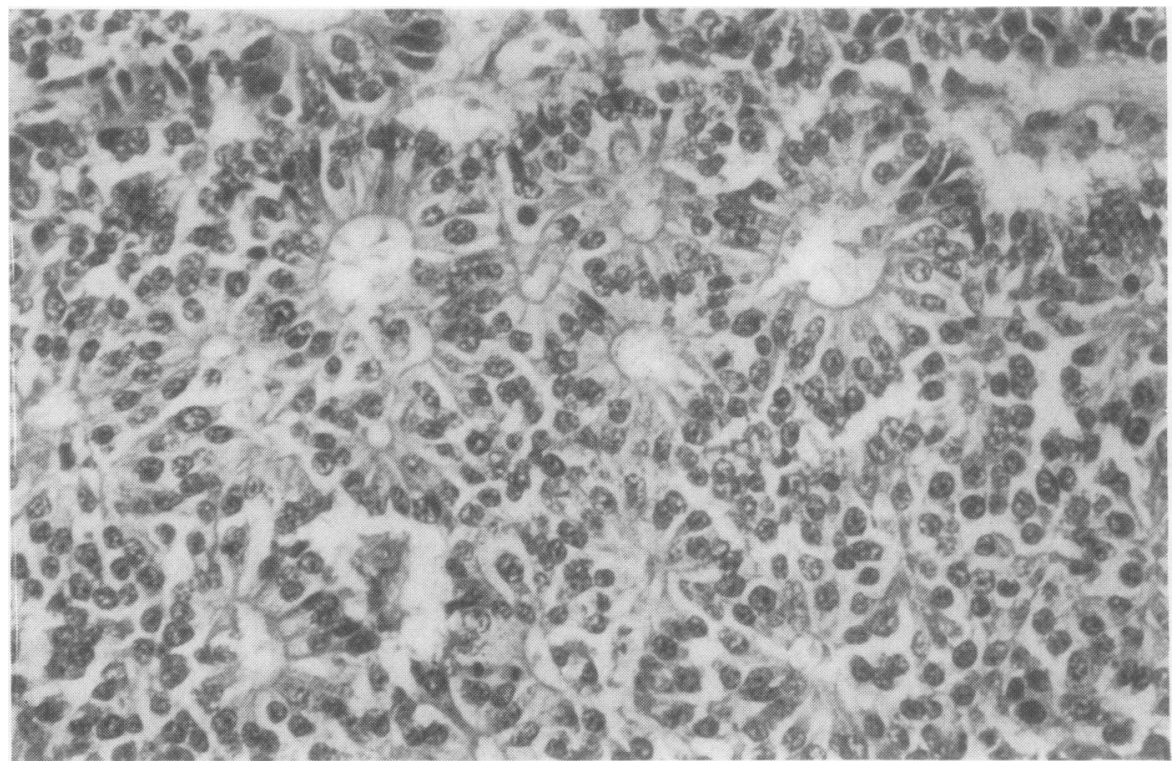

FIG. 4. Tumour showing rosette formations $(H$ and $E \times 40)$.

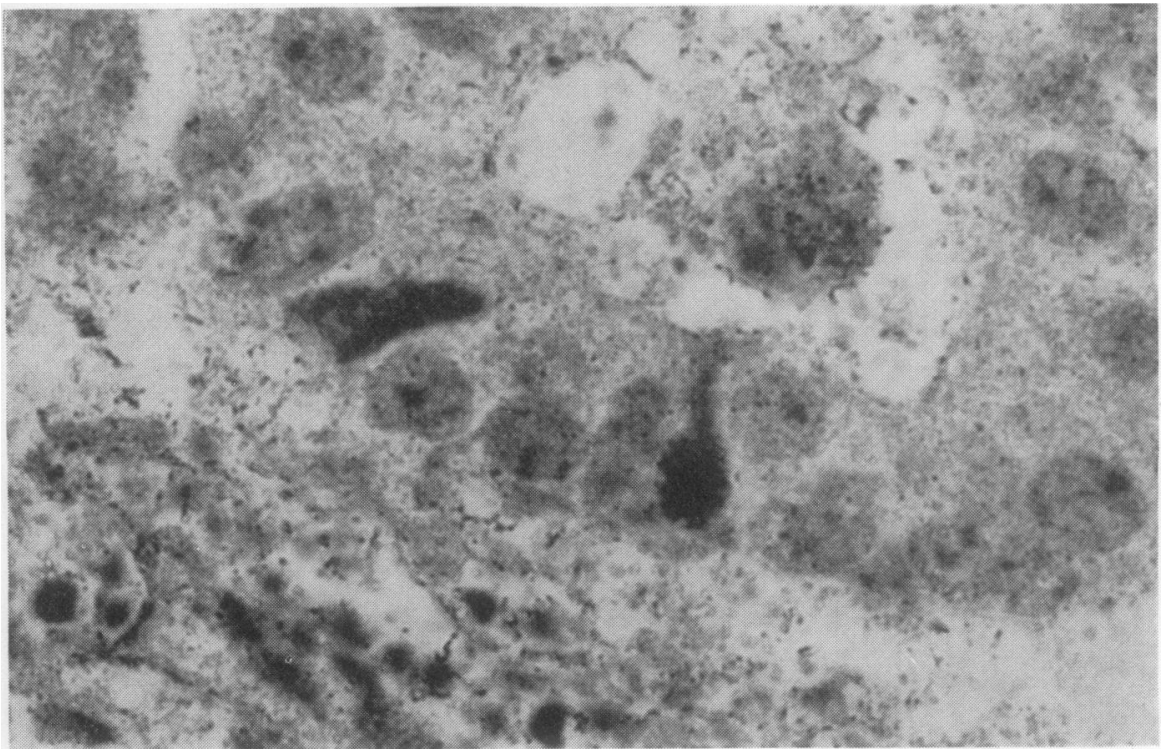

FIG. 5. Tumour cells containing argyrophil granules (Bodian $\times 400$ ). 
acetate showed no evidence of mucin secretion or cilia on the luminal border of the rosettes. Autolytic changes had destroyed most cytoplasmic structures and although membrane-bound granules were present they could not be certainly identified.

\section{DISCUSSION}

In many important respects the case reported here corresponds to those described by Rosai and Higa (1972) as mediastinal endocrine neoplasm of probable thymic origin related to carcinoid tumour. Our case was male, as were 14 of the 16 described by them, though ours was older than any of theirs at diagnosis (present case, age 72; age range of previously reported cases, 21 to 66 ). Histologically the presence of true rosettes and of argyrophil cells is typical, as is the location in the anterior mediastinum. In our case we have been able to confirm absolutely the situation of the tumour in the thymus by retrograde thymic venography. No bronchial connection was discovered at necropsy, nor was there evidence of a primary tumour in the lung, pancreas or elsewhere. On histological and anatomical grounds, therefore, the likelihood that the tumour arose elsewhere than in the thymus would seem remote.

Clinically, however, the present case showed a number of features not previously associated with this type of tumour. Except for three cases Rosai and Higa reported separately (Rosai, Higa, and Davie, 1972) associated with multiple endocrine adenomatosis (MEA), their cases were either asymptomatic, the tumour being discovered on routine chest radiographs, or were accompanied by local symptoms only. In contrast, there was in our case a variety of systemic features of the non-metastatic type. He had polyarthritis as the initial manifestation, and clubbing of the fingers. $\mathrm{He}$ also had clinical evidence suggesting the presence of a mild proximal myopathy, namely, difficulty in raising himself from the sitting position and weakness of the deltoids, left triceps, and trunk and thigh muscles. A probable mild peripheral neuropathy was evidenced by symptoms of paraesthesiae of the fingers and of difficulty in holding cups, and by the finding of blunting of light touch sensation of the fingertips. These features are well known in association with other types of tumour, for example, oat-cell carcinoma of the lung. However, it has been suggested that this also is derived from a secretory cell possibly of neuro-ectodermal origin (Pearse, 1969; Weichert, 1970). There was no suggestion clinically or at necropsy of MEA or of hormone production. The latter is often associated with an epithelial type of thymoma, which may sometimes be of similar endocrine origin (Macadam and Vetters, 1969), although this is not always established (Lemon, Fine, Grasso, and Kinsell, 1966).

Our patient also had an intermittent dysrhythmia and a systolic ejection-type murmur originating from the pulmonary region. At necropsy the pulmonary valve was normal, and no definite cause for the murmur was identified, but it may have been caused by extrinsic pressure on the pulmonary outflow tract by the large tumour mass in the anterior mediastinum.

The aggressive behaviour of the tumour in our case, with only a four-month survival from diagnosis and the presence at death of multiple metastases, resembles that in the previously reported cases of carcinoid tumour of the thymus with MEA (Rosai et al., 1972). These authors were unable to find any histological distinction between these cases and cases unassociated with MEA (Rosai and Higa, 1972), although the latter ran a much more benign course.

The poor response of the tumour to radiotherapy (Rosai and Higa, 1972) is again confirmed in this case and contrasts with the typically dramatic response of other thymic tumours to this form of treatment (Skeggs, 1968). As surgical excision appears to be the only form of effective treatment (Rosai and Higa, 1972), it would seem important to obtain tissue for histological diagnosis in any similar case by thoracotomy or by mediastinoscopy and biopsy (Skeggs, 1973). Only the most serious risk factors should contraindicate such a course of action.

\section{REFERENCES}

Lemon, F. C., Fine, M. B., Grasso, S. G., and Kinsell, L. W. (1966). ACTH-like activity in a thymoma associated with gonadal dysgenesis. Journal of Clinical Endocrinology, 26, 1.

Macadam, R. F. and Vetters, J. M. (1969). Fine structural evidence for hormone secretion by a human thymic tumour. Journal of Clinical Pathology, 22, 407.

Pearse, A. G. E. (1969). The cytochemistry and ultrastructure of polypeptide hormone-producing cells of the APUD series, and the embryologic, physiologic, and pathologic implication of the concept. Journal of Histochemistry and Cytochemistry, 17, 303.

Rosai, J. and Higa, E. (1970). Primary mediastinal carcinoid tumour: clinicopathological study of 7 cases. Laboratory Investigation, 22, 508.

and - (1972). Mediastinal endocrine neoplasm, of probable thymic origin, related to carcinoid tumour. Clinicopathologic study of 8 cases. Cancer, 29, 1061. 
and Davie, J. (1972). Mediastinal endocrine neoplasm in patients with multiple endocrine adenomatosis. A previously unrecognized association. Cancer, 29, 1075.

Skeggs, D. B. L. (1968). Radiotherapy of thymic tumours in myasthenia gravis. Proceedings of the Royal Society of Medicine, 61, 760. (1973). Complications associated with the radiotherapy of thymic tumours. Proceedings of the Royal Society of Medicine, 66, 155.
Weichert, R. F. (1970). The neural ectodermal orig产 of the peptide-secreting endocrine gland American Journal of Medicine, 49, 232.

Requests for reprints to: Dr. R. M. Lowenthat MRC Leukaemia Unit, Royal Postgraduate Medica School, Du Cane Road, London W12 0HS. 